\title{
Photonics-based tunable 1-50 GHz RF transmitter on silicon chip
}

\author{
F. Falconi ${ }^{1}$, C. Porzi ${ }^{1}$, M. Malik ${ }^{1}$, F. Scotti ${ }^{2}$, A. Malacarne ${ }^{2}$, P. Ghelfi ${ }^{2}$, A. Bogoni ${ }^{1,2}$ \\ ${ }^{I}$ TeCIP - Scuola Superiore Sant'Anna, Via Moruzzi 1, 56124 Pisa, Italy \\ ${ }^{2}$ CNIT - National Photonics Labs, Via Moruzzi 1, 56124 Pisa, Italy \\ e-mail:antonella.bogoni@cnit.it
}

\begin{abstract}
The paper presents an innovative tunable software-defined RF transmitter based on integrated photonics, able to work in $1-50 \mathrm{GHz}$ with very fast tuning. The system has been characterized and verified in a communication scenario
\end{abstract}

OCIS codes: 250.0250 Optoelectronics; 280.5600 Radar;

\section{Introduction}

Software-defined ultra-wideband (UWB) and tunable radio frequency (RF) transmitters able to operate in the whole $\mathrm{RF}$ domain (beyond $40 \mathrm{GHz}$ ), find application in many civilian and military fields, as in communications for 6G [1] [2], radars/imaging systems and electronic warfare (EW) [3]. In the communication field, various UWB transmitters, that operate in the $3.1-10.6 \mathrm{GHz}$ unlicensed band, have been presented due to their benefits in term of high data rate, robustness to propagation fading, interference rejection, and coexistence with narrow band systems [2]. In advanced UWB radar systems (able to detect the time-frequency signature of the targets), transmitters must digitally generate a wide variety of waveforms in different frequency ranges, tailored to the target type of interest [4].

Considering the military field, UWB communication offers additional advantages such as superior obstacle penetration, undercover operation, resistance to jamming, etc. [1]. High dynamic range and wideband performance in the $0-40 \mathrm{GHz}$ range and beyond are also required in EW, where such a kind of transmitters can be used as jammers for UWB communication links and radars [3]. Currently, the jammers work in specific RF bands, especially in the range 1-6 GHz, 5-18GHz, while jammers working at higher frequencies are still under definition.

For all the above-mentioned applications, UWB transmitter must be able to provide frequency agility, flexibility, and reconfigurability, with optimized size, weight, and power consumption (SWaP). Unfortunately, the lack of tunability in state-of-the-art electronic and microwave technology (e.g., tunable RF filters and tunable mixer) does not allow to implement this kind of systems, and the coverage of the UWB spectral range of interest is usually obtained through the use of several narrow band transmitters, thus leading to high SWaP.

Photonic techniques applied in RF UWB transmitters can support achieving the desired features. In the last few years, in fact, photonics has been demonstrating attractive features for microwave applications, as extremely wide operating and instantaneous bandwidth, tunable filtering, tunable photonics-based microwave mixing with very high port-to-port isolation, and intrinsic immunity to electromagnetic interferences [5]. An UWB photonics-based transmitter for radio-over-fiber systems has been proposed in [6]. It presents an operating BW higher than $60 \mathrm{GHz}$, but it makes use of a widely tunable synthesizer to operate at different frequencies. A filter-free photonics-based bulk solution is also presented in [7] working up to $26 \mathrm{GHz}$.

In this paper, an innovative tunable RF transmitter enabled by integrated photonics and implemented on chip by CMOS-compatible technology is presented; working in the range $0.5-50 \mathrm{GHz}$ (L, S, C, X, Ku, K, Ka bands) with very fast tuning $(<100 \mu \mathrm{s})$, suitable for $6 \mathrm{G}$ systems and counteracting radar.

\section{Photonics-based RF transmitter design and fabrication}

Fig. 1 (left) shows the architecture of the tunable photonics-based RF transmitter. The generation of the tunable desired RF signal is based on the beating in a photodiode of an optical replica of the waveform to be transmitted, with a tunable optical carrier at the spectral distance equal to the desired RF. The optical replica of the waveform is obtained through the modulation of an input laser in single-sideband carrier-suppression (SSB-CS) format. This modulation is obtained by means of a dual-parallel Mach-Zehnder modulator (MZM) driven by the digitallygenerated waveform converted in the analog domain at intermediate frequency $\mathrm{f}_{\mathrm{IF}}$ by means of a digital-to-analog converter (DAC). This way, the optical single-sideband signal represents a replica of the original waveform. A tunable optical carrier is obtained by the tunable selection of one the optical lines out of an optical comb. The comb generator is composed of a phase modulator (PM) driven by an electrical clock at frequency $\mathrm{f}_{\text {clk }}$, modulating a second copy of the input laser. Then, a tunable optical filter selects the desired optical line at desired RF distance from the sideband. The tunable optical filter, that is one of the key components of the scheme, is made of a cascade 
of multiple-cavity phase-shifted Bragg gratings [8]. In particular, $4^{\text {th }}$-order filters are used, in which five Bragg gratings are separated by four grating "defects" a quarter wavelength-long, where the phase is carefully controlled by means of micro-heaters above the defects themselves. The selected optical line and the sideband are finally injected into the $40 \mathrm{GHz}$ Germanium photodiode where they beat and generate an electrical replica of the waveform at the desired RF. This signal is then sent to a RF front-end before the transmission.

The photonic scheme (excluding the laser) has been implemented on-chip as a photonic integrated circuit (PIC) exploiting the silicon-on-insulator (SOI) photonic platform. The fabricated PIC is shown in the inset of Fig. 1(left).

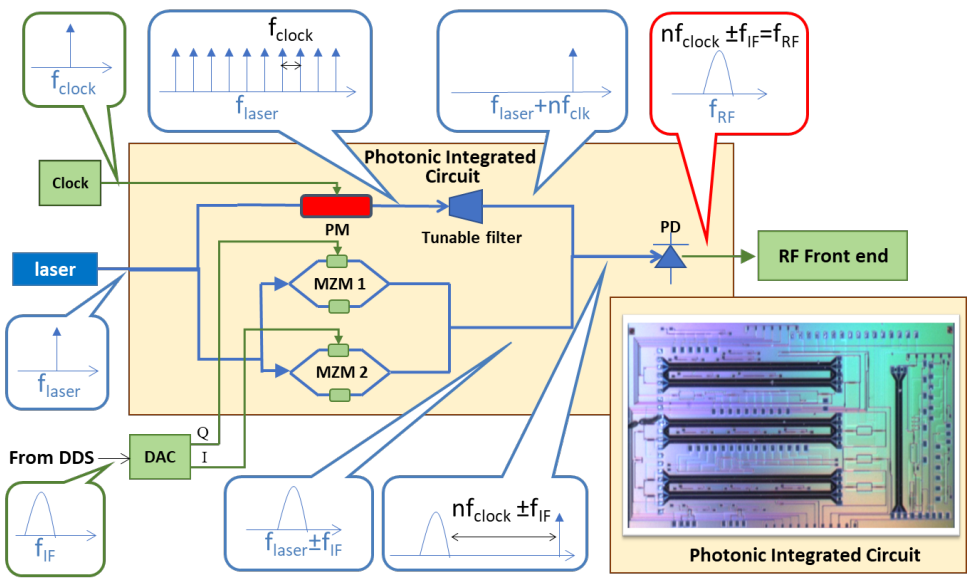

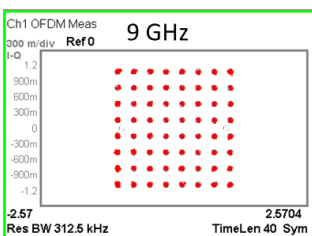

$E V M=33,5 \mathrm{~dB}$

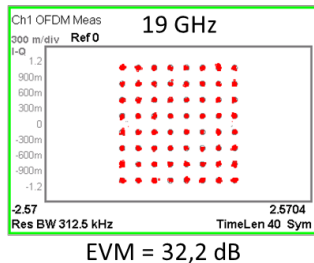

$\mathrm{EVM}=32,2 \mathrm{~dB}$

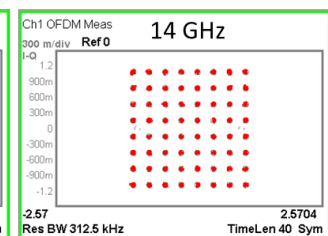

$\mathrm{EVM}=33,8 \mathrm{~dB}$

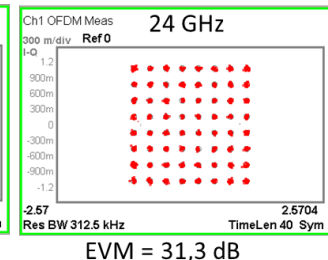

Fig. 1. (left): Scheme of the photonics-based RF transmitter. DAC: digital-to-analog converter; PM: phase modulator; MZM: Mach-Zehnder modulator; PD: photodiode, Inset: the PIC; (right): 64QAM constellations generated at 9, 14, 19, $24 \mathrm{GHz}$ (instrument limited to $26 \mathrm{GHz}$ ).

\section{Photonics-based RF transmitter design, fabrication and testing}

The implemented chip is characterized, first at level of single components thanks to monitor points specifically inserted in the photonic circuit, and then at system level. The PIC is placed on a thermo-electric cooler that, thanks to a thermistor, maintains the PIC temperature with an accuracy of $0.01^{\circ} \mathrm{C}$. The optical signals are injected and extracted from the PIC with an array of fibers, while the electrical signals are injected or extracted from the chip through an RF multiprobe.

We report here the characterization of the tunable optical filter, that is one of the most important and challenging components. Fig. 2 (left) reports the filter transmission spectrum for a specific central frequency. It is worth to notice that its $3 \mathrm{~dB}$ bandwidth is as narrow as $<4 \mathrm{GHz}$, while the $30 \mathrm{~dB}$ bandwidth is $<10 \mathrm{GHz}$. Consequently, the filter is able to select the desired line of the optical comb for $\mathrm{f}_{\text {clk }}$ (i.e. the comb frequency) $\geq 5 \mathrm{GHz}$ with an extinction ratio $>30 \mathrm{~dB}$. The filter range tunability is also verified in the whole C-band, confirming the optical filter is suitable for generating RF signals $>40 \mathrm{GHz}$.

The filter tunability speed determines the RF transmitter tunability speed. This parameter is measured by varying the control signal of one of the 4 micro-heaters with a step function, so as to be able to move the filter from a condition of misalignment with the test signal, to a condition of perfect alignment (see Figure 2-right). By observing the time variation of optical power at the output of the filter, we have a precise measure of the tunability speed of the filter. The $10 \%-90 \%$ transient is less than $200 \mu \mathrm{s}$ when the filter cools to move to higher frequencies, while it is faster (below $60 \mu \mathrm{s}$ ) when the filter is heated to move it in the opposite direction. This is because cooling is a slower process, with the chip having to dissipate excess heat. Heat dissipation is known to occur with slow dynamics, as the phenomenon of thermal conduction involves a large portion of the PIC to disperse the heat. The temperature increase is faster, as the thermal conduction involves only the part of the optical circuit near the heaters.

Fig. 3 (left) shows the RF carriers generated in $\mathrm{C}, \mathrm{X}, \mathrm{Ku}, \mathrm{K}, \mathrm{Ka}$ and $\mathrm{V}$ bands all overlapped in the same graph in the case of $\mathrm{f}_{\text {clk }}=5 \mathrm{GHz}$ and a sinusoidal waveform at $\mathrm{f}_{\mathrm{IF}}=4 \mathrm{GHz}$ (used as upper or lower SSB). In all cases, the extinction ratio is $>25 \mathrm{~dB}$ thanks to the excellent extinction ratio of the filter and of the SSB-SC, both $>35 \mathrm{~dB}$. Then, the RF transmitter is characterized in terms of phase noise. Fig. 3 (right) shows the phase noise curves of the RF signal generated at high frequencies $(35,40,45,50 \mathrm{GHz})$, where the highest noise level is expected. The comparison with the reference clock signal at $5 \mathrm{GHz}$ (in our case, a synthesizer) is also reported. Phase noise curves are measured in a range up to $10 \mathrm{MHz}$ around the nominal frequency. This range is limited by the instrument. The noise of the signal generated is also analyzed up to $50 \mathrm{GHz}$, even though it cannot be displayed because it is outside the range of the electrical spectrum analyzer. It can be seen that the performance does not significantly degrade as the frequency increases. From the phase noise curves the temporal jitter of the signals is then calculated, which 
confirms the substantially similar performance for all the radio frequencies generated, and a value always lower than $200 \mathrm{fs}$.
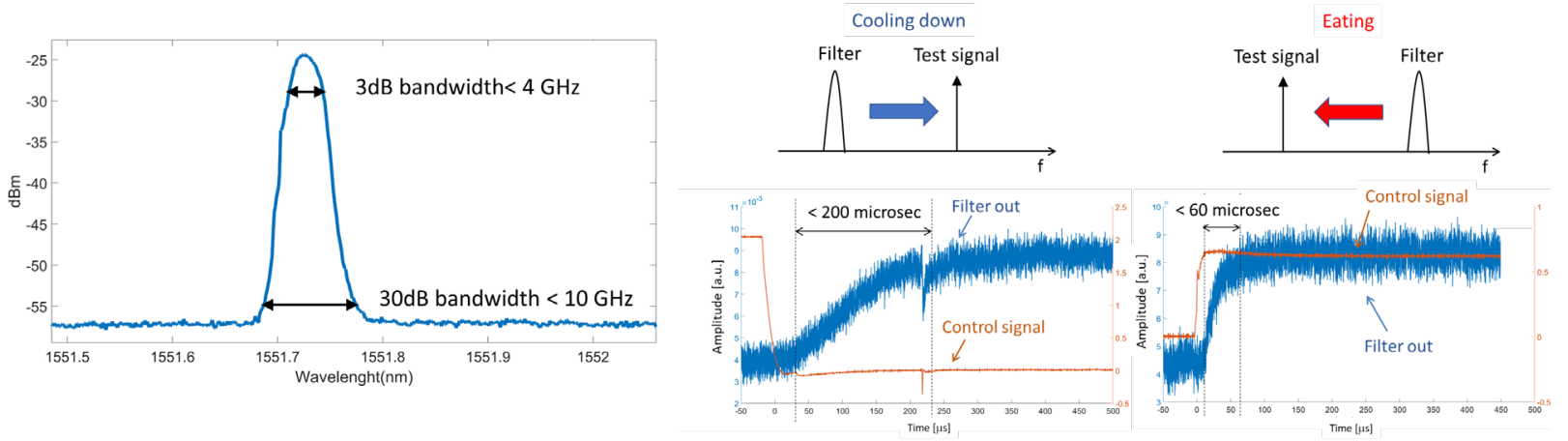

Fig. 3 (left): tunable optical filter transmission; (right) Time behavior of the signal at the output of the filter as it passes from a condition of misalignment with the signal to one of alignment, in the case of movement towards higher frequencies for cooling (left) and movement towards lower frequencies for heating (right).
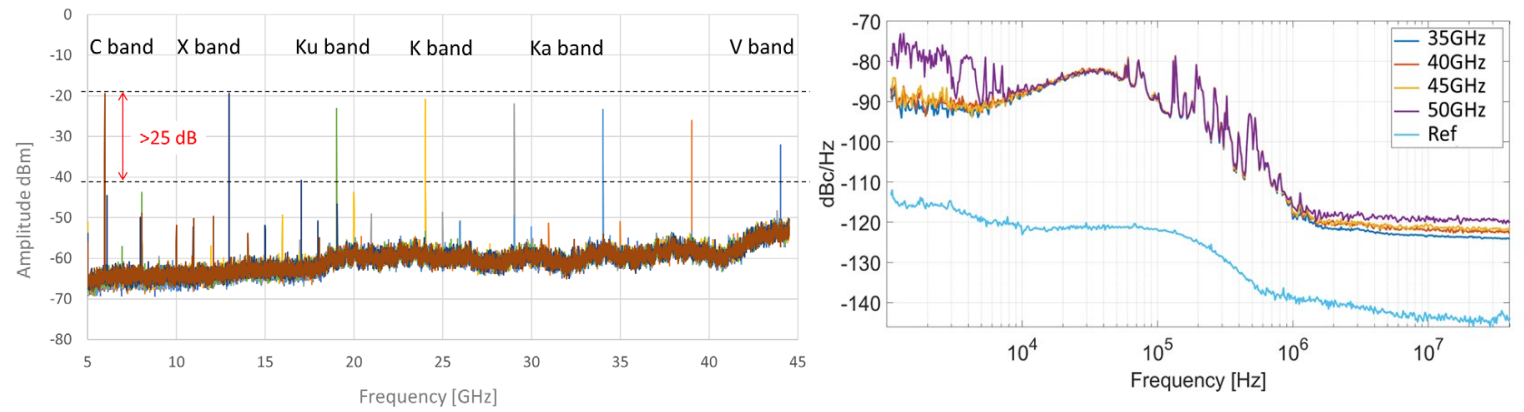

Fig. 3. (left): RF carriers generated in $\mathrm{C}, \mathrm{X}, \mathrm{Ku}, \mathrm{K}, \mathrm{Ka}$ and $\mathrm{V}$ bands; (right): phase noise curves of the RF signal generated for different values of the carrier.

The photonics-based transmitter is also characterized in terms of its ability to generate a real communication signal, as shown in Figure 1 (right). The data signal is generated at an intermediate frequency of $4.063 \mathrm{GHz}$ and modulated with 64QAM format and a bandwidth of $20 \mathrm{MHz}$. The clock signal remains at 5GHz. The signal generated by the beating in the photodiode is then analyzed using a vector signal analyzer. The performance is evaluated by measuring the error vector magnitude (EVM). The analysis is limited by the bandwidth of the vector signal analyzer $<26 \mathrm{GHz}$. The EVM measurement detects variations $<3 \mathrm{~dB}$ as the frequency varies, and a penalty with respect to the reference signal (i.e. obtained without tunable up-conversion) of less than $4 \mathrm{~dB}$ in all cases.

The conversion efficiency of the RF transmitter, defined as the ratio between the power of the RF output signal and the IF input signal, is measured $=-66 \mathrm{~dB}$. This low value is one of the major issues of the system. It can be improved with advancements in technology to reduce losses in the photonic circuit and by introducing optical amplification stages that are possible with a hybrid integration platform. Another technological advance consists in reducing the $\mathrm{V} \pi$ voltage necessary for modulators to transfer intermediate frequency signals to the optical domain, and reduce their optical attenuation and nonlinearities.

The activity has been partially supported by DISTURB project funded by Italian Ministry of Defense.

\section{References}

[1] White Paper, "Cisco Visual Networking Index: Global Mobile Data Traffic Forecast Update 2017-2022” (2019).

[2] Moe Z. Win, et al., "History and Applications of UWB", Proceedings of the IEEE, Vol. 97, No. 2, February 2009.

[3] Michael Frater and Michael J. Ryan "Electronic Warfare for the Digitized Battlefield", Artech House, January 2001.

[4] James D. Taylor, “Advanced Ultrawideband Radar: Signals, Targets, and Applications", CRC Press, December 14, 2016.

[5] Ghelfi, P, Laghezza F., Bogoni A., "Photonics for Radar Networks and Electronic Warfare Systems" Editors: IET SciTech Book Series: Radar, Antennas and Electromagnetics, 2019, ISBN: 9781785613760

[6] O Omomukuyo, et al., "Performance Evaluation of a 60-ghz Multi-band Ofdm (mb-ofdm) Ultra-wideband Radio-over-fibre System”, IEEE Journal on Selected Areas in Communications, 2011.

[7] D. Onori et al., "A Software-defined and Filter-free 0 26.5 GHz Ultra wideband RF Transmitter Enabled by Photonics", EuRAD 2017

[8] C. Porzi, G. J. Sharp, M. Sorel and A. Bogoni, "Silicon Photonics High-Order Distributed Feedback Resonators Filters," in IEEE Journal of Quantum Electronics, vol. 56, no. 1, pp. 1-9, Feb. 2020, 